\title{
On the Comparison of Gauss and Hybrid Methods and their Application to Calculation of Definite Integrals
}

\author{
MEHRİBAN İMANOVA \\ Computational mathematics, Baku State University \\ Institute of Control Systems named after Academician A. Huseynov, \\ Executive Director, Science Development Foundation \\ under the President of the Republic of Azerbaijan \\ Baku, AZERBAIJAN \\ mehriban.imanova@sdf.gov.az
}

\begin{abstract}
As is known there is the wide class of methods for calculation of the definite integrals constructed by the well-known scientists as Newton, Gauss, Chebyshev, Cotes, Simpson, Krylov and etc. It seems that to receive a new result in this area is impossible.

The aim of this work is the applied some general form of hybrid methods to computation of definite integral and compares that with the Gauss method. The generalization of the Gauss quadrature formula have been fulfilled in two directions. One of these directions is the using of the implicit methods and the other is the using of the advanced (forward-jumping) methods. Here have compared these methods by shown its advantages and disadvantages in the results of which have recommended to use the implicit method with the special structure. And also are constructed methods, which have applied to calculation of the definite integral with the symmetric bounders. As is known, one of the popular methods for calculation of the definite integrals with the symmetric bounders is the Chebyshev method. Therefore, here have defined some relations between of the above mentioned methods. For the application constructed, here methods are defined the necessary conditions for its convergence. The receive results have illustrated by calculation the values for some model integral using the methods with the degree $p \leq 8$.
\end{abstract}

Key-Words: - The initial-value problem for ODE, definite integral, Gauss method, multistep hybrid method, forward-jumping method.

Received: October 18, 2019. Revised: February 13, 2020. Accepted: March 6, 2020. Published: March 27, 2020.

\section{Introduction}

As is known the first quadrature formula has been constructed by Newton. The other authors have developed the construction of quadrature formula by using different interpolation polynomials. By choosing of the interpolation polynomials the scientists constructed the quadrature methods with a higher order of exactness. There are numerous works dedicated to investigation of the definite integrals (see for example [1]-[12]).For the investigation of the definite integrals here have used subintervals method and constructed algorithms by using of which, one can compare results received here with the known.

Let us consider computation of the definite integral $I=\int_{a}^{b} f(x) d x$ which has been replaced by the computing of the values of some function. For example, let us consider the following function:
Here the sufficiently smooth function $f(x)$ is defined on the $[a, b]$.

From here receive that

$$
I=y(b)
$$

and

$$
\begin{gathered}
y\left(x_{i+1}\right)=y\left(x_{i}\right)+\int_{x_{i}}^{x_{i+1}} f(x) d x,(i=0,1, \ldots, n-1), \\
x_{0}=a, x_{n}=b .
\end{gathered}
$$

By the $x_{i}(i=0,1, . ., n)$ are denoted the nodal points. If we compare this formula with the composite known corresponding formulas, then receive that the formula has proposed here has some preference. By using this formula have constructed the methods which are the same with the methods applied to solving of the initial-value problem for the ODE. Thus the calculation of the definite integral has reduced to calculation of the integral on 
the subinterval which has the length equal to $h$. For the calculation of the values of the definite integrals, here proposed to use some of the hybrid methods, which have applied to solve the initialvalue problem for the ODE of the first order. Taking into account that Gauss quadrature formula one of the more exact for the calculation of the values of the definite integrals, here give some comparison of Gauss method with the proposed here methods. Note that, in some cases, determined the maximal exactness of the proposed method and constructed some concrete methods with the degree $p \leq 8$. It is known that in the application of the Riemann integrals, most popular is the definite integrals with the symmetric bounders. There are many methods for calculation of the next integral $\int_{-a}^{a} f(x) d x$. Among them, we can be noted the Gauss and Chebyshev methods. Remark that the mentioned integral in basically have investigated for the value $a=1$, which is related with the good studies of the linear independent systems on the segment $[-1,1]$. By using the wide application of the integrals with the symmetric bounders in the fields of mechanics, biology, communication, nuclear physics, earthquakes and natural periodic seismic processes and etc., here consider the application of the hybrid methods to the calculation of the definite integrals with the symmetric bounders(see for example [13][20]. The way proposed here has used some properties of the definite integrals with the symmetric bounders.

\section{On the application of the hybrid methods to calculation of simple definite integral}

For the calculation of the following integral

$$
I=\int_{a}^{b} f(x) d x,
$$

let us use the next function:

$$
y(x)=\int_{a}^{x} f(s) d s, a \leq x \leq b .
$$

It is obvious that $y(b)=I$. By taking into account that the correlation (2) is equality, therefore from (2) one can write:

$$
y^{\prime}(x)=f(x) .
$$

It is not difficult to understand that the equality (2) and ordinary differential equation (3) is equivalent.
Thus the calculation of the integral (2) we replace by solving of the ODE of the first order.

For the finding, of the unique solution of the equation (3) let us in the equality (2) put $x=a$. Then receive:

$$
y(a)=0 .
$$

The problem (3) and (4) is the initial-value problem, which fundamentally investigated by many authors (see for example [21]-[29]).

As is known one of the popular methods for solving problem (3) and (4) is the multistep method with the constant coefficients, which can be written as:

$$
\sum_{i=0}^{k} \alpha_{i} y_{n+i}=h \sum_{i=0}^{k} \beta_{i} f_{n+i} .
$$

Here $\alpha_{i}, \beta_{i}(i=0,1, \ldots, k)$ are some real numbers, $\alpha_{k} \neq 0$ and $y_{m}=y\left(x_{m}\right), f_{m}=f\left(x_{m}\right),(m=0,1,2, \ldots)$. Suppose that the coefficients $\alpha_{i}, \beta_{i}(i=0,1, \ldots, k)$ satisfy the conditions $\mathrm{A}, \mathrm{B}$, and $\mathrm{C}$ from the work [21].

The last time for solving the problem (3) and (4) the scientists proposed to use the hybrid method, which in simple form can be written as:

$$
\sum_{i=0}^{k} \alpha_{i} y_{n+i}=h \sum_{i=0}^{k} \gamma_{i} f_{n+i+v_{i}},\left(\left|v_{i}\right|<1 ; i=0,1, \ldots, k\right) .
$$

It is known that all of the methods have its advantages and disadvantages. Therefore let us use the linear combination of the methods (5) and (6) for construction of the new methods with the best properties. For this, let us consider the investigation the following methods:

$$
\begin{array}{r}
\sum_{i=0}^{k} \alpha_{i} y_{n+i}=h \sum_{i=0}^{k} \beta_{i} f_{n+i}+h \sum_{i=0}^{k} \gamma_{i} f_{n+i+v_{i}}, \\
\left(\left|v_{i}\right|<1 ; i=0,1, \ldots, k\right) .
\end{array}
$$

Usually, the points $x_{i+v_{i}}=x_{0}+\left(i+v_{i}\right) h$ call as the hybrid points. If we apply this method to the calculation of the integral (1), then receive:

$$
\int_{a}^{b} f(x) d x=h \sum_{i=0}^{n} A_{i} f_{i}+h \sum_{i=0}^{n} B_{i} f_{i+n+v_{i}} .
$$

If in this equality put $B_{i}=0,(i=0,1, \ldots, n)$, then receive the Newton-Cotes method and if put $A_{i}=0,(i=0,1, \ldots, n)$, then one can be received the Gauss or Chebyshev method. From here receives that the investigation of the method (8) is perspective. To show this, let us consider the comparison of the method (8), Gauss method and Chebyshev method in the next section by the calculation of the integral with the symmetric bounders. Therefore put $A_{i}=0$ and denote by $x_{i}=x_{i}+v_{i} h,(i=0,1, \ldots, n)$. 
In this case to determine the coefficients $B_{i}$ and nodal points $X_{i}(i=0,1, \ldots, n)$ of the Gauss method one can use the following nonlinear system of algebraic equations (see [7,p. 190-193]):

$$
\begin{aligned}
& B_{0}+B_{1}+\ldots+B_{n}=\mu_{0}, \\
& B_{0} x_{0}+B_{1} x_{1}+\ldots+B_{n} x_{1}=\mu_{1}, \\
& B_{0} x_{0}^{m}+B_{1} x_{1}^{m}+\ldots+B_{n} x_{1}^{m}=\mu_{m} .
\end{aligned}
$$

Here $\mu_{j}(j=0,1, . ., m)$ are the known. If the system (9) has the solution, then by using that one can construct the method of type (8) (Gauss method). But the investigation of this system is difficult, therefore in [7, p.191] proposed to use roots of the polynomial:

$$
\omega(x)=\left(x-x_{0}\right)\left(x-x_{1}\right) \ldots\left(x-x_{n}\right)
$$

For the determination of the values of the variables $A_{i}, B_{i}, v_{i}(i=0,1, \ldots, n)$, here propose the other way. For the described this way let us apply the method (8) to solve the problem (3) and (4). Then receive:

$$
\sum_{i=0}^{k} \alpha_{i} y_{n+i}=h \sum_{i=0}^{k} \beta_{i} y_{n+i}^{\prime}+h \sum_{i=0}^{k} \gamma_{i} y_{n+i+v_{i}}^{\prime}
$$

It is not difficult to prove that to get the method (10) with the degree $p$, the satisfying of its coefficients of the following conditions are necessary and sufficient:

$$
\begin{gathered}
\sum_{i=0}^{k} \alpha_{i}=0 ; \sum_{i=0}^{k}\left(i \alpha_{i}-\beta_{i}-\gamma_{i}\right)=0 \\
\sum_{i=0}^{k}\left(\frac{i^{2}}{2 !} \alpha_{i}-i \beta_{i}-\left(i+v_{i}\right) \gamma_{i}\right)=0 \\
\sum_{i=0}^{k}\left(\frac{i^{p}}{p !} \alpha_{i}-\frac{i^{p-1}}{(p-1) !} \beta_{i}-\frac{\left(i+v_{i}\right)^{p-1}}{(p-1) !} \gamma_{i}\right)=0 .
\end{gathered}
$$

For the investigation of the system (14) let us consider the case $k=1$. Then to determine the values of the unknown receive the following system:

$$
\begin{aligned}
& \gamma_{0}+\gamma_{1}=1, \\
& v_{0} \gamma_{0}+\left(1+v_{1}\right) \beta_{1}=1 / 2, \\
& v_{0}^{2} \gamma_{0}+\left(1+v_{1}\right)^{2} \beta_{1}=1 / 3, \\
& v_{0}^{3} \gamma_{0}+\left(1+v_{1}\right)^{3} \beta_{1}=1 / 4 .
\end{aligned}
$$

Let us note that from the conditions $k=1$ follows that $\alpha_{0}+\alpha_{1}=0$ and is known that for the finding from equality (6) the value $y_{n+k}$ usually take $\alpha_{k}=1$. Therefore receive $\alpha_{1}=-\alpha_{0}=1$. By taking these values in the system (14) receive the system of (15). By solving this system receive:

$$
v_{0}^{2}-v_{0}+1 / 6=0
$$

And the unknown $v_{1}$ can be defined from the condition:

$$
v_{0}+v_{1}=0 \text {. }
$$

Thus by solving the system (15) receive that

$$
\begin{aligned}
& \gamma_{0}=\gamma_{1}=1 / 2, \\
& v_{0}=-v_{1}=1 / 2-\sqrt{3} / 6 .
\end{aligned}
$$

In this case method (6) can be written as:

$$
\begin{aligned}
y_{n+1} & =y_{n}+h\left(f\left(x_{n}+h / 2-\sqrt{3} h / 6\right)+\right. \\
& \left.+f\left(x_{n}+h / 2+\sqrt{3} h / 6\right)\right) / 2 .
\end{aligned}
$$

If in the system of (15) added more than one equation, then receive that the receiving system has not any solution. Thus receive that the method (16) is unique and there is not the method with the degree $p>4$.

Now let us construct the more exact methods by using the formula (7). For this put $k=1$. Then for the determination of the values of the coefficients for the hybrid method receive the following system:

$$
\begin{gathered}
\beta_{1}+\beta_{0}+\gamma_{1}+\gamma_{0}=1, \\
\beta_{1}+l_{1}^{j} \gamma_{1}+l_{0}^{j} \gamma_{0}=1 /(j+1) \\
\left(j=1,2,3,4,5 ; l_{i}=i+v_{i} ; i=0,1\right) .
\end{gathered}
$$

By using the solution of this system one can construct the stable methods with the degree $p \leq 6$. The method with the degree $p=6$ can be written as:

$$
\begin{gathered}
y_{i+1}=y_{i}+h\left(f_{i}+f_{i+1}\right) / 12+ \\
+5 h\left(f_{i+1 / 2-\beta}+f_{i+1 / 2+\beta}\right) / 12 ; \\
\beta=\sqrt{5} / 10 .
\end{gathered}
$$

For the construction the stable methods with the degree $p>6$, let us consider the case $k=2$ in the system of (14), then to define the system of algebraic equations for the finding of the values of the coefficient, receive the following system:

$\beta_{2}+\beta_{1}+\beta_{0}+\gamma_{2}+\gamma_{1}+\gamma_{0}=2^{j+1} \alpha_{2}+\alpha_{1}$,

$2^{j} \beta_{2}+\beta_{1}+l_{2}^{j} \gamma_{2}+l_{1}^{j} \gamma_{1}+l_{0}^{j} \gamma_{0}=\left(2^{j+1} \alpha_{2}+\alpha_{1}\right) /(j+1)$

$\left(j=1,2,3,4,5,6,7 ; l_{i}=i+v_{i}\right)$.

By using the solution of this system have constructed the following method when $\alpha_{2}=-\alpha_{0}=1, \alpha_{1}=0$ :

$$
\begin{gathered}
y_{i+2}=y_{i}+h\left(9 f_{i+2}+64 f_{i+1}+9 f_{i}\right) / 90+ \\
+h\left(49 f_{i+1+\sqrt{21} / 7}+49 f_{i+1-\sqrt{21} / 7}\right) / 90,
\end{gathered}
$$


here $l_{2}=1+\sqrt{21} / 14 ; l_{0}=1-\sqrt{21} / 14$. This method is stable and has the degree $p=8$. Remark that this can be rewritten as the one-step method. For this let us change $h_{\text {by the }} h / 2$, then receive:

$$
\begin{aligned}
y_{i+1}= & y_{i}+h\left(9 f_{i+1}+64 f_{i+1 / 2}+9 f_{i}\right) / 180+ \\
& +h\left(49 f_{i+l_{2} / 2}+49 f_{i+l_{0} / 2}\right) / 180 .
\end{aligned}
$$

This is one-step method with the degree $p=8$ and in the application which to calculation of definite integrals are not arising any difficulties.

\section{Computation of the definite integrals by using multistep hybrid methods of the forward-jumping type}

As was noted above the aim of this work is the construction of more exact stable multistep methods. For this aim, in this section present to use the hybrid methods of the forward-jumping type.

It is known, that classic multistep method with constant coefficients has been constructed in two forms one of them is the method (5) and the second can be written as follows:

$$
\sum_{i=0}^{k-m} \alpha_{i} y_{n+i}=h \sum_{i=0}^{k} \gamma_{i} f_{n+i}(i=0,1, \ldots, N-k), \quad m>0,
$$

here $y_{m}(m \geq 0)$ is approximately values of the solution of the equation (3), the continuous function $f(x)$ is given, but $\alpha_{i}(i=0,1, \ldots, k-m)$ and $\gamma_{i}(i=0,1, \ldots, k)$ are real numbers and $\alpha_{k-m} \neq 0$. This condition related with that the solution of the finitedifference equation (20) is the value $y_{n+k-m}$. Coefficient for which must to satisfy the conditions $\alpha_{k-m} \neq 0$. And by using the method constructed to found the value of $y_{n+k-m}$.

Let us note that the class of the methods (5) and (20) is not intersection. In formally for the value $m=0$ from the method (20) follows the method (5). But it is not correct. Forward-jumping method received in the case $\quad\left|\alpha_{k}\right|+\left|\alpha_{k-1}\right|+\ldots+\left|\alpha_{k-m+1}\right| \neq 0 \quad$ and $\left|\beta_{k}\right|+\left|\beta_{k-1}\right|+\ldots+\left|\beta_{k-m+1}\right| \neq 0$. Therefore the methods of the type (5) and (20) are independent object for investigation.

Note that the multistep method (5) was fundamentally investigated by Dahlquist (see [21]) and he prove that if the method (5) has the degree $p$ then $p \leq 2 k$ is hold. But if the method (5) is stable and has the degree $p$ then $p \leq 2[k / 2]+2$ and there are the stable methods with the degree $P_{\max }$ for all values of $k$.
Definition 1. The integer $p$ is called as the degree for the method (5) if the following is hold:

$$
\sum_{i=0}^{k}\left(\alpha_{i} y(x+i h)-h \beta_{i} y^{\prime}(x+i h)\right)=O\left(h^{p+1}\right) .
$$

The definition of stability can be given as follows: Definition 2. The method (5) calls as stable if all roots of the polynomial $\rho(\lambda)=\alpha_{k} \lambda^{k}+\alpha_{k-1} \lambda^{k-1}+\ldots+\alpha_{1} \lambda+\alpha_{0}$ lie in the unit circle on the boundary of which there is no multiply root. The notation degree and stable are defined for the method (20) by the same way. The method (20) fundamentally was investigated (see for example [22]) and prove that if the method (20) has the degree of $p$ then $p \leq 2 k-m$ is hold and if the method (20) is stable then $p \leq k+1+m(k \geq 3 m)$. From here receive that the method (5) is more exact for unstable methods, but the method (20) is more exact for stable methods. In the class of methods (20) there are stable symmetric methods. These methods are effective in the application of them to solve the integral equations with the symmetric bounders (see [17]).

As was noted in the previous section one of the modern problems for Computational Mathematics is the construction of the method with the high exactness. And it is known that scientists have constructed the methods on intersection of the Runge-Kutta and Adams methods which is called as the hybrid methods. And prove that hybrid methods are more exact than Runge-Kutta and Adams methods. In previous section has been shown that there are stable hybrid methods which have the degree $p \geq 2 k$. By using the advantages of the hybrid methods have constructed and investigated the following multistep hybrid methods of forwardjumping type:

$$
\sum_{i=0}^{k-m} \alpha_{i} y_{n+i}=h \sum_{i=0}^{k} \beta_{i} f_{n+i}+h \sum_{i=0}^{k} \gamma_{i} F_{n+i+y_{i}} \quad\left(v_{i} \mid<1, i=0,1, \ldots, k\right), m>0 .
$$

For the investigation of this method let us begin from the determination of necessary condition imposed on the coefficients of the method (21) which can be written as follows:

The coefficients $\alpha_{i}(i=0,1, \ldots, k-m), \beta_{i}, \gamma_{i}, v_{i}(i=0,1, \ldots, k)$ are some real numbers and $\alpha_{k-m} \neq 0$.

The polynomials

$$
\rho(\lambda)=\sum_{i=0}^{k-m} \alpha_{i} \lambda^{i} ; \sigma(\lambda)=h \sum_{i=0}^{k} \beta_{i} \lambda^{i} ; \gamma(\lambda)=h \sum_{i=0}^{k} \gamma_{i} \lambda^{i+v_{i}}
$$

have no common factor different from constant. The conditions $\sigma(1)+\gamma(1) \neq 0$ and $p \geq 1$ are hold.

The necessity of the condition $\alpha_{k-m} \neq 0$ have described above. 
Therefore the condition $A$ is evident. Let us consider proof of the necessity of the condition $B$.

Assume the contrary and denote by $\varphi(\lambda)_{\text {the }}$ common factor of these polynomials. Let us consider the following shift operator:

$$
E^{l} y(x)=y(x+l h) \text {. }
$$

By using this operator the equality (21) let us write in the following form:

$$
\rho(E) y_{n}-h \sigma(E) y_{n}^{\prime}-h \gamma(E) y_{n}^{\prime}=0
$$

As is known from the theory of finite-difference equations that for the existence of the unique solution of the finite difference equation (22) should be known initial values $y_{0}, y_{1}, \ldots, y_{k-1}$ of the function $y(x)$. It is not difficult to understand that the equation (22) can be rewrite

in the following form:

$$
\phi(E)\left(\rho_{1}(E) y_{n}-h \sigma_{1}(E) y_{n}^{\prime}-h \gamma_{1}(E) y_{n}^{\prime}\right)=0,
$$

here

$$
\begin{aligned}
& \rho_{1}(\lambda)=\rho(\lambda) / \phi(\lambda), \\
& \sigma_{1}(\lambda)=v(\lambda) / \phi(\lambda), \\
& \gamma_{1}(\lambda)=\gamma(\lambda) / \phi(\lambda) .
\end{aligned}
$$

From here receive that

$$
\rho_{1}(E) y_{n}-h \sigma_{1}(E) y_{n}^{\prime}-h \gamma_{1}(E) y_{n}^{\prime}=0,
$$

since $\phi(\lambda) \neq$ const .

It is evident that the order of difference equation (24) is at most $k-1$, so that $\phi(\lambda) \neq$ const. Therefore, taking into account the equivalent of equation (23), (24) we get that equation (22) has a unique solution if the $k-1$ initial values are given, that contradict general theory of finite-difference equations. From here receive that the condition $\mathrm{B}$ is hold.

Now let us prove that if method (21) converges, then the condition $\mathrm{C}$ is hold. For this aim the equality (22) can be rewritten in the following form:

$$
\rho(E) y(x)=O(h),
$$

here $x=x_{0}+n h$-fixed point.

Pass to the limit here for $h \rightarrow 0$ receive that:

$$
\rho(1)=0 \text {. }
$$

This is necessary condition for the convergence of the method (21). By using the shift operator and the necessary condition of convergence for the multistep method we can write the relation (22) in the form:

$$
\begin{aligned}
& \rho_{1}(E)\left(y_{i}-y_{i-1}\right)-h \sigma(E) y_{i}^{\prime}-h \gamma(E) y_{i}^{\prime}=0 \\
& \left(\rho_{1}(\lambda)=\rho(\lambda) /(\lambda-1)\right) .
\end{aligned}
$$

Here we change the parameter $i$ from 1 to $n$, and summing the obtained equalities. Then we get

$$
\rho_{1}(E)\left(y_{n}-y_{0}\right)-\sigma(E) h \sum_{j=1}^{n} y_{j}^{\prime}-\gamma(E) h \sum_{j=1}^{n} y_{j}^{\prime}=0 .
$$

Passing to limit as $h \rightarrow 0$ and to use the properties of the integral sum, we have:

$$
\rho_{1}(1)\left(y(x)-y_{0}\right)=(\sigma(1)+\gamma(1)) \int_{x_{0}}^{x} f(s) d s,
$$

Here $x=x_{0}+n h$ is a fixed point.

By using the equality

$$
y(x)=y_{0}+\int_{x_{0}}^{x} f(s) d s
$$

in the equality (29) receive the following:

$$
\rho_{1}(1)=\sigma(1)+\gamma(1) .
$$

By using the following

$$
\rho_{1}(\lambda)=\rho(\lambda) /(\lambda-1) \text { and } \lim _{\lambda \rightarrow 1} \rho_{1}(\lambda)=\rho_{1}(1)
$$

in the equality of (30) receive:

$$
\rho_{1}^{\prime}(1)=\sigma(1)+\gamma(1) \text {. }
$$

By this description receive that $\rho_{1}(1)=0$ and $\rho_{1}^{\prime}(1)=\sigma(1)+\gamma(1)$, that coincide with the first two equations of system (14) where from it follows that $p \geq 1$. Note that if $\sigma(1)+\gamma(1)=0$, we get $\rho_{1}^{\prime}(1)=0$. Hence it follows that method (21) is not stable. But it is known that the stability is a necessary and sufficient condition for the convergence of the method (5). Consequently the condition $\mathrm{C}$ holds. Thus we proved that the restrictions $\mathrm{A}, \mathrm{B}, \mathrm{C}$ are natural and suppose that they holds everywhere.

As was noted above, if method (20) is stable and has the degree $\mathrm{p}$, then $p \leq 4 k+2-m$. The method with the degree $p=6$, obtained for $k=1$ and $m=0$ is stable. For $k=2$ one can construct stable methods with the degree $p=8$ and $p=9$.

Let us note that method (19) has been constructed as the partial case of the method (7) for the value $k=2$. The properties of the Gauss quadrature formula are hold for the method (19). The hybrid points for this method are located as symmetric to the center for the bounders of the integral and corresponding coefficients are the same. But these properties are not satisfy to the method having the degree $p=9$. Therefore here basically have used the method (19): And now let us consider the construction of the stable methods of the type (20) and (21). For this aim, have been proposed some ways to find the values of the coefficients for the method (20) and (21). By using the similarity (7) and (21) to find the values of the coefficients for the method (21) one can be used the following system of algebraic equations:

$$
\begin{array}{r}
\sum_{i=0}^{k-m} \alpha_{i}=0 ; \sum_{i=0}^{k}\left(\beta+\gamma_{i}\right)=\sum_{i=0}^{k-m} \dot{\alpha}_{i} ; \\
\sum_{i=0}^{k}\left(i \beta_{i}+\left(i+v_{i}\right) \gamma_{i}\right)=\sum_{i=0}^{k-m} \frac{i^{2}}{2 !} \alpha_{i} ;
\end{array}
$$




$$
\sum_{i=0}^{k}\left(\frac{i^{l-1}}{(l-1) !} \beta_{i}+\frac{\left(i+v_{i}\right)^{l-1}}{(l-1) !} \gamma_{i}\right)=\sum_{i=0}^{k-m} \frac{i^{l}}{l !} \alpha_{i} \quad(l=3,4, \ldots, p) .
$$

If in this system consider the case $\gamma_{i}=0 \quad(i=0,1, \ldots, k)$ then from the system (31) receive the linear system of the algebraic equation to find the values of the coefficients for the method (20). Note that the system (31) is nonlinear. Let us consider the case $m=1$. In this case receive that $k-1>0$. It is follow that $k \geq 2$. If put $k=2$, then receive the following method:

$$
y_{n+1}=y+h\left(5 f_{n}+8 f_{n+1}-f_{n+2}\right) / 12 .
$$

This forward-jumping method is stable and has the degree $p=3$. But for the value $k=2$ from the method (5) one can receive the stable method with the degree $p=4$ which called as the Simpson's method. As was noted above if the method (5) is stable and has the degree $p$, then for $k=3$ the degree

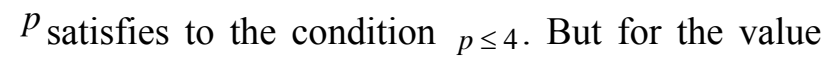
$k=3$ in the class of methods of the type (20) there are stable methods with the degree $p=5$, which can be written as:

$$
\begin{aligned}
y_{n+2}= & \left(11 y_{n}+8 y_{n+1}\right) / 19+ \\
& h\left(10 f_{n}+57 f_{n+1}+24 f_{n+2}-f_{n+3}\right) / 57 .
\end{aligned}
$$

From here receive that the stable forward-jumping methods can be more exact than the implicit methods so as the method (33) from the class of forward-jumping methods.

Note that one of disadvantages of the hybrid methods to contain the calculation of the values of the function $y(x)$ at the hybrid points. Therefore some specialists in construction of these methods proposed to use only rational mesh points instead of hybrid points. Therefore let us construct the hybrid method of the type (21) by using rational mesh points. For this let us put $m=1, k=2$ and $\alpha_{1}=-\alpha_{0}=1, v_{0}=1 / 2, v_{1}=0, v_{2}=-1 / 2$. In this case the amount of the unknowns will be decreases in result receive that the amount of the unknowns equal to 5 . Therefore has been constructed the stable method by using the solution of the linear system (31) can be written as:

$$
\begin{aligned}
y_{n+1}=y_{n} & +h\left(29 f_{n}+24 f_{n+1}-f_{n+2}\right) / 180 \\
& +h\left(62 f_{n+1 / 2}+2 f_{n+3 / 2}\right) / 90 .
\end{aligned}
$$

This method is stable and has the degree $p=5$.

If compare the methods (32)-(34), then receive that the methods (32) and (34) can be taken as symmetric. Because the value $y_{n+1}$ find itself between the value $y_{n}$ and $y_{n+2}$.

Remark. Application of the constructed here methods depends from the function participated in the right hand of the differential equation. If the differential equation given as $y^{\prime}=f(x, y)$, then in using of the hybrid method arise some difficulties related on the calculation of the values $y\left(x_{n}+\left(i+v_{i}\right) h\right)$

.Singularity in the case when the value $v_{i}(i \geq 0)$ is irrational number. But in the application of hybrid method to compute the definite integrals these questions aren't arise. To illustrate this here applied the method (19) to compute the definite integral given in the first exercise. Results have been tabulated in the tabl 1.

\section{Applications of the hybrid methods to computation of the definite integrals with the symmetric bounders}

Now let us consider the calculation of the values for the definite integrals with the symmetric bounders by using the hybrid methods. For this aim consider the following equality:

$$
I_{s}=\int_{-1}^{1} f(s) d s=\int_{0}^{1}(f(s)+f(-s)) d s .
$$

One of the popular methods for the calculation of the integral (35) is the Gauss method, which can be written as the following (see for example [4, p. 468]):

$$
I=f\left(-\frac{1}{\sqrt{3}}\right)+f\left(\frac{1}{\sqrt{3}}\right) .
$$

If apply this method to the computation of the integral $\int_{-1}^{0} f(s) d s+\int_{0}^{1} f(s) d s$ to the determination of its values, then receives:

$$
I_{s}=\left(f\left(\frac{1}{\sqrt{3}}\right)+f\left(\frac{1}{\sqrt{3}}\right)\right) / 2+\left(f\left(\frac{1}{\sqrt{3}}\right)+f\left(\frac{-1}{\sqrt{3}}\right)\right) / 2,
$$

which concede with the value of (36).

If we compare the formulas (37) and (36) then receive that these results are the same. But now let us reduce calculation of the integral (35) to solving the initial-value problem for the ODE of the first order. For this aim let us consider the following function:

$$
y(x)=\int_{-x}^{x} f(s) d s, 0 \leq x \leq 1 .
$$

From here one can receive the following problem:

$$
y^{\prime}=f(x)+f(-x), y(0)=0 .
$$

For solving this problem let us consider the simple case and applied Euler's method to solving of the problem (39). Then receive:

$$
y_{n+1}=y_{n}+h f_{n}+h f_{-n} .
$$

From the theory of numerical methods which have applied to solve the initial-value problem for the ODE is known that the results received by the 
implicit methods are more exact than the results received by explicit methods. But in the application of these methods to the calculation of the values of definite integrals, there are free from these properties (the properties of explicit and implicit). Still in this case the implicit methods give the more exact results. To show this let us compare method (40) with the next trapezoidal rule:

$$
\begin{aligned}
& \int_{-x_{1}}^{x_{1}} f(s) d s=\int_{0}^{x_{1}}(f(s)+f(-s)) d s= \\
& =h\left(f\left(x_{1}\right)+f\left(-x_{1}\right)+2 f(0)\right) / 2
\end{aligned}
$$

and by using the method (40) receive:

$$
\int_{-x_{1}}^{x_{1}} f(s) d s=2 h f(0)
$$

As is known the method (41) is more exact than the explicit Euler's method. And these methods with the point of view to fill out of computational works are the same. Therefore to replace the calculation of definite integral to solving of the initial-value problem for the ODE is expedient.

Now let us consider the application of the hybrid methods to calculation definite integrals with the symmetric bounders. For this aim let us consider the calculation of the definite integral in the subsegment $\left[x_{0}, x_{k}\right]$. In this case receive:

$$
y_{k}=\int_{x_{0}}^{x_{k}} f(s) d s \approx h \sum_{i=0}^{k} \beta_{i} f_{i} .
$$

By using the above-described way we can calculate the values of the definite integrals with the symmetric bounders by using the methods which have applied to the calculation of the values of the integrals with the nonsymmetrical bounders. In this case, receive the following:

$$
\begin{gathered}
\int_{-x}^{x} f(s) d s=h \sum_{i=0}^{k} \beta_{i}\left(f_{n+i}+f_{-n-i}\right)+ \\
\quad+h \sum_{i=0}^{k} \gamma_{i}\left(f_{n+i+v_{i}}+f_{-n-i-v_{i}}\right), \\
\text { here } f_{-m}=f\left(-x_{m}\right),(m \geq 0) .
\end{gathered}
$$

Let us consider the partial case and put $k=1$. Then receive the following:

$$
\begin{gathered}
y_{i+1}=y_{i}+h\left(f\left(x_{i}+(3-\sqrt{3}) h / 6\right)+\right. \\
\left.+f\left(x_{i+1}+(3-\sqrt{3}) h / 6\right)\right) / 2+ \\
+h\left(f\left(-x_{i}-(3-\sqrt{3}) h / 6\right)+\right. \\
\left.f\left(-x_{i+1}+(3-\sqrt{3}) h / 6\right)\right) / 2,(i=0,1, . ., n) .
\end{gathered}
$$

This method has the degree $p=4$ and in the application of which are not arise any difficulties, if don't count the calculation of the function of $f(x)$ at the irrational points. Noted that the hybrid methods can be constructed without the participation of the irrational points, but in this case the values of the degree for these methods are decreases. As is follows from here the methods are constructed in the previous section can be applied to the calculation of the values of the definite integrals with the symmetric bounders.

Note that in the case $k=1$ one can construct a more exact method than the method of (42). For example the following:

$$
\begin{gathered}
y_{i+1}=y_{i}+h\left(f\left(x_{i}\right)+f\left(x_{i+1}\right)\right) / 12+ \\
+h\left(f\left(x_{i}+(1-2 \beta) h / 2\right)+\right. \\
\left.+f\left(x_{i+1}+(1+2 \beta) h / 2\right)\right) / 12+ \\
+h\left(f\left(-x_{i}\right)+f\left(-x_{i+1}\right)\right) / 12+ \\
+h\left(f\left(-x_{i}-(1-2 \beta) h / 2\right)+\right. \\
\left.+f\left(-x_{i+1}-(1+2 \beta) h / 2\right)\right) / 12, \beta=\sqrt{5} / 10 .
\end{gathered}
$$

This method is stable and has the degree $p=6$. This method contains two parts. In the first part we have used the mesh points $x_{i}=x_{0}+i h(i \geq 0)$ and in the second part have used the hybrid points in the form

\begin{tabular}{|c|c|c|c|c|c|c|}
\hline \multirow{3}{*}{$\begin{array}{l}\text { Var } \\
\text { iabl } \\
\mathrm{e}\end{array}$} & \multicolumn{6}{|c|}{ Step size $h=0,1$} \\
\hline & \multicolumn{3}{|c|}{$\lambda=1$} & \multicolumn{3}{|c|}{$\lambda=5$} \\
\hline & $f(s)$ & $f(-s)$ & $f(s)+f(-s)$ & $f(s)$ & $f(-s)$ & $f(s)+f(-s)$ \\
\hline 0.1 & $2.43 \mathrm{E}-9$ & $2.20 \mathrm{E}-9$ & $2.31 \mathrm{E}-10$ & $9.31 \mathrm{E}-6$ & $5.64 \mathrm{E}-6$ & $3.66 \mathrm{E}-6$ \\
\hline 0.4 & $1.13 \mathrm{E}-8$ & $7.62 \mathrm{E}-9$ & $3.75 \mathrm{E}-9$ & $9.17 \mathrm{E}-5$ & $1.24 \mathrm{E}-5$ & $7.92 \mathrm{E}-5$ \\
\hline 0.7 & $2.34 \mathrm{E}-8$ & $1.16 \mathrm{E}-8$ & $1.18 \mathrm{E}-8$ & $4.60 \mathrm{E}-4$ & $1.39 \mathrm{E}-5$ & $4.47 \mathrm{E}-4$ \\
\hline 1.0 & $3.97 \mathrm{E}-8$ & $1.46 \mathrm{E}-8$ & $2.51 \mathrm{E}-8$ & $2.11 \mathrm{E}-3$ & $1.42 \mathrm{E}-5$ & $2.109 \mathrm{E}-3$ \\
\hline
\end{tabular}
$x_{i}+(1 \pm 2 \beta) h / 2,(i \geq 0)$.

For the illustration of the received here results let us consider the application of methods (42) to solving of some model definite integrals.

For the illustration of the results, received here let us to consider application of the method (16) to calculation of the following definite integrals:

1. $\lambda \int_{0}^{1} \exp (\lambda s) d s$.

Corresponding problem is

$y^{\prime}(x)=\lambda \exp (\lambda x), y(0)=0$.

2. $\lambda \int_{-1}^{1} \exp (\lambda s) d s$.

Corresponding problem is

$y^{\prime}(x)=\lambda(\exp (\lambda x)+\exp (-\lambda x)), \quad y(0)=0$.

Table 1 Results of example 1

\begin{tabular}{|c|l|l|l|l|}
\hline \multirow{2}{*}{$\begin{array}{c}\text { Vari } \\
\text { able } \\
x\end{array}$} & \multicolumn{3}{|l}{ Step size $h=0,1$} \\
\cline { 2 - 5 } & $\lambda=1$ & $\lambda=5$ & $\lambda=-1$ & $\lambda=-5$ \\
\hline 0.1 & $2.43 \mathrm{E}-9$ & $9.31 \mathrm{E}-6$ & $2.20 \mathrm{E}-9$ & $5.64 \mathrm{E}-6$ \\
\hline 0.4 & $1.13 \mathrm{E}-8$ & $9.17 \mathrm{E}-5$ & $7.62 \mathrm{E}-9$ & $1.24 \mathrm{E}-5$ \\
\hline 0.7 & $2.34 \mathrm{E}-8$ & $4.60 \mathrm{E}-4$ & $1.16 \mathrm{E}-8$ & $1.39 \mathrm{E}-5$ \\
\hline 1.0 & $3.97 \mathrm{E}-8$ & $2.11 \mathrm{E}-3$ & $1.46 \mathrm{E}-8$ & $1.42 \mathrm{E}-5$ \\
\hline
\end{tabular}

Table 2 Results of example 2

As is follows from the contents of these tables the error for the values $\lambda>0$ increases, but for the 
values $\lambda<0$ decreases. These properties coincide with the corresponding theory of numerical methods. By the results receiving in solving of the example 2 one can to assert that the error receiving in the solving of the example 2 is not worse than the error receiving in solving of the example 1.

\section{Conclusion}

As is known to study the initial-value problem for ODE may realize by using the Volterra integral equations. For example, prove of the theorem about on the existence and uniqueness of the solution of that problem, construction Euler's method and etc. But here we used the contrary way and have considered to the application of the methods to the calculation of the definite integrals, which have constructed to solve the initial-value problem for the ODE. As is known one of the more exact methods for calculation of the definite integrals is the Gauss method.

Therefore, here have compares the hybrid and Gauss methods and determines their intersection parts. By using this way constructed the more exact methods for calculation of the definite integrals, which have used the hybrid points. And constructed some concrete hybrid methods, which have applied to calculation some model definite integrals.

Let us note that the receive results in the calculation of the model definite integrals have shown the advantages of the methods proposed here.

\section{References:}

[1] Burden R.L, Douglas, Faires Cengege Lerning, J. 2001. 850 pp., 7-th edition.

[2] Quarteroni A., Saccar R., Saleri F. Numerical Mathematics, 2007, Springer.

[3] Krylov A.N. 1950. Lectures on approximate calculations. Moscow, Gocteh-izdat.

[4] Krylov V.I., Bobkov V.V., Monastyrovy, P.I. Computational methods of higher mathematics. Minsk: The Higher School, 1972, 584 p.

[5] Mamedov J.J. Computational methods, 1978, $304 \mathrm{pp}$.

[6] Bahvalov N.S. Lapin A.V. Chizhonkov E.V. Numerical methods in problems and examples , Moscow, Vysshaya Shkola, 2000, p.190.

[7] Verzhbitsky V.M. Numerical Methods Linear Algebra and Linear Equations, M.: Vysshaya Shkola, 2000, 297.

[8] Atkinson K.E. A survey of numerical methods for solving nonlinear integral equations. Journal of integral equations and applications, 1992, No 1, v.4, p.15-46.
[9] Stroud A. H., Secrest D. Gaussian Quadrature Formulas. 1966, IX, 369 S. London. Prentice Hall, Inc. Preis geb.

[10] Marchuk G., Shaidurov V. Improving the accuracy of solutions of difference schemes. Moscow, Nauka, 1979, 319p.

[11] Nikolsky S.M. Quadrature formulas. Moscow, Science, 1979, $256 \mathrm{p}$.

[12] Aliev T.A. Digital Noise Monitoring of Defect, Origin Series Springer, London, 2007, 235 p.

[13] Aliev T.A., Abbasov A.M., Guluyev G.A., Pashayev F.G., Sattarova U.E. System of robust noise monitoring of anomalous seismic process, Soil Dynamics and Earthquake Engineering, 53, 2013, 11-26.

[14] Michael P. Fitz Analog Communication Theory, The Ohio State University, 2001, p. 200

[15] Fatyullayeva L.F. The limiting state of $a$ multilayer nonlinearly lactic eccentric ring, Journal "Mechanics of composite Materials", New York, vol 43, 2007, No.6, pp. 513-520.

[16] Mehdiyeva G., Imanova M., Ibrahimov V. Some refinement of the notion of symmetry for the Volterra integral equations and the construction of symmetrical methods to solve them, Journal of Computational and Applied Mathematics, 2016, 306, 1-9.

[17] Mehdiyeva G., Imanova M., Ibrahimov V. An Application of Mathematical Methods for Solving of Scientific Problems, British Journal of Applied Science \& Technology - Volume 14,2016 , issue 2, 1-15.

[18] Mehdiyeva G.Yu., Imanova M.N., Ibrahimov V.R. General Theory of the Application of Multistep Methods to Calculation of the Energy of Signals, Wireless Communications, Networking and Applications, Volume 348 of the series Lecture Notes in Electrical Engineering, 2016, pp. 1047-1056.

[19] Mehdiyeva G., Imanova M., Ibrahimov V. A way for finding the relation between of the degree and order for multistep method used to applied to solving of the Volterra integrodifferential equation WSEAS Transactions on Mathematics, Volume 17, 2018, 155-161.

[20] Mehdiyeva G., Imanova M., Ibrahimov V. On the application of multistep methods to solving some problems of communication. Proceedings of the International Conference on Numerical Analysis and Applied Mathematics (ICNAAM2014) AIP Conf. Proc. 1648, 2015, AIP Publishing LLC, 850050-1-850050-5. 
[21] Dahlquist G. Convergence and stability in the numerical integration of ordinary differential equations, 1956, Math.Scand, No.4, 33-53.

[22] Mehdiyeva G.Yu., Ibrahimov V.R. On the research of multi-step methods with constant coefficients. 2013, Monograph, Lambert. acad. publ.

[23] Butcher J.C. A modified multistep method for the numerical integration of ordinary differential equations. J. Assoc. Comput. Math., 1965, v.12, 124-135.

[24] Gear C.S. Hybrid methods for initial value problems in ordinary differential equations. SIAM, J. Numer. Anal. v. 2, 1965, 69-86.

[25] Mehdiyeva G., Ibrahimov V., Imanova M. On One Application of Hybrid Methods for Solving Volterra Integral Equations, World Academy of Science, engineering and Technology, Dubai, 2012, 809-813.

[26] Mehdiyeva G., Imanova M., Ibrahimov V. On the construction of the forward-jumping method and its application to solving of the Volterra integral equations with symmetric boundaries WSEAS Transactions on Mathematics, Volume 16, 2017, 295-302.

[27] Gupta G.K. A polynomial representation of hybrid methods for solving ordinary differential equations. Mathematics of comp., volume 33, 1979, number 148, 1251-1256.

[28] Mehdiyeva G., Imanova M., Ibrahimov V. Guliyeva A. One a way for constructing hybrid methods with the constant coefficients and their applied, Alloys and Experimental Mechanics, IOP Conf. Series: Materials Science and Engineering 225, 2017, 012042, 1-6. 